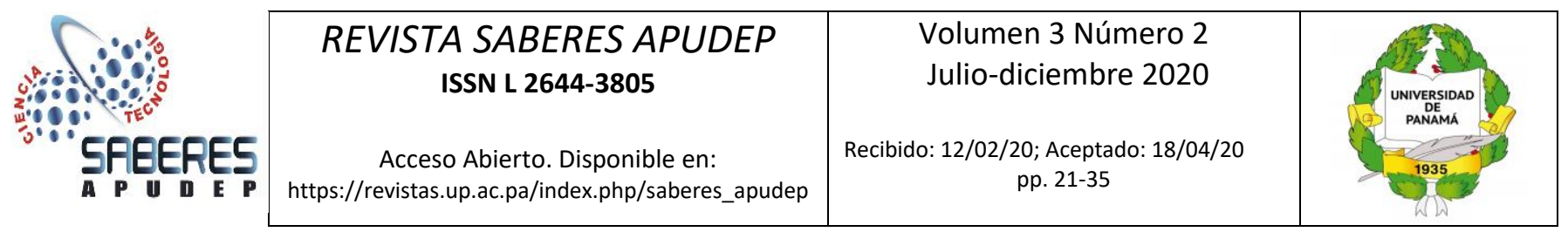

\title{
DELIMITACIÓN ENTRE AUTORÍA Y PARTICIPACIÓN DELICTIVA
}

\author{
BORDERLINE BETWEEN PERPETRATORSHIP AND CRIMINAL PARTICIPATION
}

\section{Orestes Arenas Nero}

\author{
Universidad de Panamá. Departamento Ciencias Penales y Criminologías. Centro Regional \\ Universitario de San Miguelito \\ profesororestes@gmail.com \\ https://orcid.org/0000-0001-5230-308
}

\section{RESUMEN}

Este artículo explicó cómo se ha entendido en la doctrina penal panameña y extranjera la delimitación entre autoría y participación. Para lograr esto se hace un recorrido por las principales teorías al respecto. Primero se analizó la teoría objetivo-formal, luego la teoría material, posteriormente la teoría subjetiva y, finalmente, la teoría del dominio del hecho. Se utilizaron técnicas de revisión bibliográfica nacional e internacional. En la misma se llegó a la conclusión, entre otras, que la teoría del dominio del hecho es la dominante en la dogmática penal, sin embargo, la teoría subjetiva no ha sido superada totalmente, por lo que mantiene un nivel de aceptación; mientras que las teorías objetivas si fueron superadas.

PALABRAS CLAVE: Delimitación, autoría, participación, Derecho Penal.

\begin{abstract}
This paper explained how the delimitation between perpetratorship and criminal participation has been understood in Panamanian and foreign criminal theory. To achieve this, a review of the main theories is made. Objective-formal theory was analyzed first, then material theory, then subjective theory, and finally, the theory of the control over. National and international bibliographic review techniques were used. In it was concluded, among others, that the theory of the control over is the dominant one in the penal theory, however, the subjective theory has not been totally overcome, so it maintains a level of acceptance; while objective theories were overcome.
\end{abstract}

KEY WORDS: Borderline, perpetratorship, Criminal Participation, Criminal Law. 


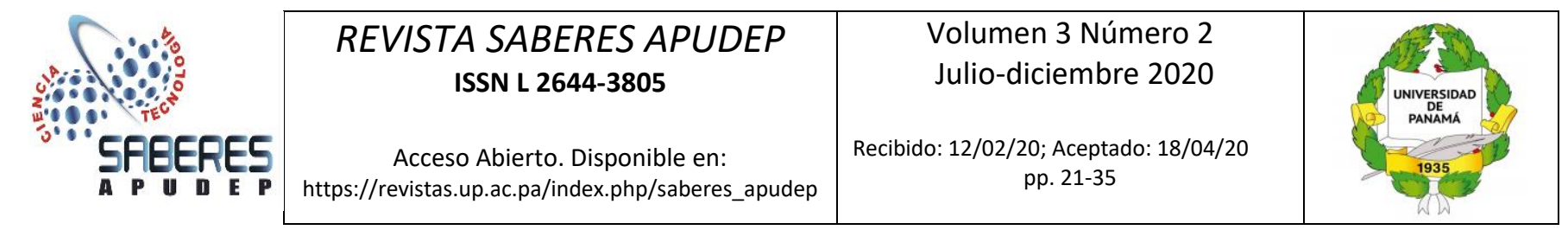

\section{INTRODUCCIÓN}

A este artículo le interesa determinar cuáles son las teorías que permiten delimitar la autoría delictiva de la participación delictiva. Esto se hace con la finalidad de conocer las distintas teorías que han permitido diferenciar al autor del partícipe. Esto se debe a la posibilidad de que todos los intervinientes en un delito no realicen actos idénticos, incluso, alejados de la descripción típica. Por ejemplo, A convence a $\mathrm{B}$ de que realice un robo. $B$ va y compra un arma en el negocio de $C$, que no sabe nada del delito. Posteriormente, $B$ va donde $D$ para que lo ayude a cometer su robo. Luego $B$ va y le pide a $E$ que lo lleve a robar y los extraiga del lugar junto con el botín. Cuando roban, B apunta con el arma mientras D desapodera. Al final B se queda con el botín y le da un pago a $D$ y a $E$. ¿Quién es autor? ¿quién es partícipe? ¿quién es impune? La respuesta a estas preguntas puede variar, dependiendo de la teoría que se siga.

En este caso, para la teoría objetivo-formal, la material-objetiva y la teoría del dominio del hecho, $B$ y $D$ serían coautores, mientras que $A$ y $E$ serían partícipes ( $A$ instigador, y $B$ cómplice). Para la teoría subjetiva $B$ sería el autor, $A$ instigador, mientras que $D$ y E serían cómplices. En todas las teorías $C$ es impune. Como se puede apreciar, frente a un mismo hecho se puede llegar a distintas conclusiones. $Y$ aquellas conclusiones parecidas son el producto de razonamientos distintos. Para la teoría objetivo-formal $B$ y $D$ son autores porque realizan la conducta descrita en el tipo penal, mientras que para la teoría del dominio del hecho, B y D son autores porque ejercieron un control sobre la realización del delito (dominio funcional).

Esta es una discusión válida, ya que es importante conocer el criterio material o normativo que permite distinguir entre la autoría y la participación delictiva. Aunque "no es fácil distinguir cuando estamos frente a autores o partícipes" (Gill, 2014). Por lo que el presente esfuerzo académico se justifica. 


\begin{tabular}{|c|c|c|}
\cline { 2 - 3 } & REVISTA SABERES APUDEP & Volumen 3 Número 2 \\
\hline
\end{tabular}

Las teorías más importantes que permiten distinguir entre autor y partícipe son: "la teoría objetivo-formal, la teoría material-objetiva, la teoría subjetiva y la teoría del dominio del hecho." (Arango, 2017). Si bien existen otras delimitaciones entre la autoría y la complicidad, como lo son "la de la conditio sine qua non, la del «dominio del hecho» O la de las "aportaciones necesarias para el resultado»" (Escrihuela, 2016). Al presente trabajo investigativo le interesa destacar las mencionadas al inicio del párrafo.

\section{DESARROLLO}

\section{TEORÍAS OBJETIVO-FORMALES}

Las primeras teorías que se explicará serán objetivo-formales, que "distinguen entre autor y partícipe sobre la base de la descripción típica." (Benítez, 2007). Estas distinguían "la cualidad del autor o de partícipe de conformidad con el aspecto exterior de los comportamientos, o sea, dependiendo si la acción se puede subsumir formalmente en el tipo penal." (Gill, 2014). Es decir, habrá autoría siempre que el interviniente realice la conducta descrita en el tipo penal, mientras que habrá participación si, intencionalmente, se da una ayuda al autor o si se le determina. Por ejemplo, $A$, que acaba de parir, le dice a su hermana $B$ que ahogue a su hijo recién nacido (hijo de A). B accede y lo ahoga. Siguiendo la concepción objetivo-formal, A sería la instigadora mientras que $B$ sería la autora, porque $B$ fue quien causó la muerte al recién nacido. Esto evidentemente va en contra de la realidad, porque $A$ tuvo mayor control de lo sucedido que $B$, quien solo ejecutó la voluntad de $A$.

Para las teorías objetivo-formales, el autor es quien realiza el "acto ejecutivo del tipo de injusto correspondiente, quien ejecuta la acción expresada por el verbo típico." (lo resaltado no es original) (Benítez, 2007). Esta concepción es la más respetuosa del principio de legalidad y de la normatividad, ya que solamente serán autores aquellos que realicen personalmente el delito. Sin embargo, esto crea un problema, 


\begin{tabular}{|c|c|c|}
\cline { 2 - 3 } & REVISTA SABERES APUDEP & Volumen 3 Número 2 \\
\hline
\end{tabular}

ya que los casos de autoría mediata serían sancionados como participación o serían impunes. Por ejemplo, A le pone balas reales al arma de una obra de teatro, y $\mathrm{B}$ dispara contra $\mathrm{C}$ pensando que son de salva, pero, al ser reales, le ocasiona la muerte. Si se sigue la teoría objetivo-formal, A no podría ser autor, porque no realizó la conducta personalmente, sino que sería partícipe, cuando a todas luces es el que ideó e hizo todo para que el delito se cometiera.

De manera muy sintética la doctora Virginia Arango señala que, según la teoría formal objetiva se considera autor "aquel que ejecutase todo o parte de una conducta tipificada en el Código Penal, por lo que cualquiera que no quedase enmarcado en este parámetro, sería considera partícipe." (Arango, 2017). En pocas palabras, quien coopere o determine intencionalmente el delito del autor, sería cómplice o instigador, mientras que aquella persona que realice el verbo rector, será autora.

Aunque se reconoce que "hoy se encuentra abandonada" (Gill, 2014). Por lo que esta forma de delimitar la autoría y la participación ha sido ampliamente superada, por no tener en cuenta la realidad y basarse únicamente en la racionalidad de la norma.

\section{TEORÍAS SUBJETIVAS}

Las teorías subjetivas se fundamentan "en la dirección de la voluntad del partícipe, es decir, distingue entre el ánimo de autor o de partícipe o entre el interés en el delito." (Gill, 2014). De lo anterior se deduce que, es autor quien tiene el animus auctoris, o, quien quiere cometer el delito para sí, mientras que es partícipe quien tenga el animus socii, o, quien quiere participar en el delito de otro.

Para las teorías subjetivas "puede ser autor quien no haya realizado la acción descrita en el tipo" (Gill, 2014). Por ejemplo, el policía A se queda con paquetes de droga obtenidos después de un allanamiento, porque su superior, $B$, le dijo que se le entregara posteriormente, lo que efectivamente sucede. 


\begin{tabular}{|c|c|c|}
\cline { 2 - 3 } & REVISTA SABERES APUDEP & Volumen 3 Número 2 \\
\hline
\end{tabular}

Siguiente el concepto subjetivo como delimitante entre autoría y participación, B es el autor del delito de posesión de drogas agravado, mientras que A es cómplice. Esta teoría contradice la realidad, ya que A debería ser considerado autor, porque él tuvo el control sobre el delito, además, realizó la acción descrita en el tipo.

De manera muy amplia se pueden fundamentar las teorías subjetivas de la siguiente manera:

"Ilo fundamental para delimitar la autoría de la participación] será aquel elemento volitivo con que actúe el sujeto, si actúa con ánimo de autor, cualquier conducta que realice con ese ánimo sea o no indispensable indicará que este actúo como autor, en cambio su actúa con ánimo de partícipe, se considerará siempre que es partícipe." (Arango, 2017)

Esta teoría se va al otro extremo. Mientras que las teorías objetivo-formales partían de la norma, estas teorías parten únicamente de la realidad subjetiva. El problema es al momento de probar quién actuaba como autor y quien lo hacía como partícipe. En otras palabras, quién quería el hecho para sí mismo, y quién lo quería para otra persona. Con base en esto, es autor "independientemente de que el aporte brindado por el sujeto sea ínfimo o no para lograr la realización del tipo." (Arango, 2017). Esto trajo como resultado concepciones como la del 'autor intelectual' que ya han sido abandonadas.

A las teorías subjetivas "dedicaron sus esfuerzos [...] sobre todo la teoría del dolo (o del dolus) y la del interés." (Roxin, 2015). Para la teoría del dolo "el autor posee una voluntad independiente y el partícipe una voluntad dependiente." (Roxin, 2015). Es decir, la voluntad del autor es autónoma y no depende de que otra persona realice algún hecho. Mientras que la voluntad del partícipe va a depender de la voluntad de otra persona, quien sería el autor. 


\begin{tabular}{|c|c|c|}
\cline { 2 - 3 } & REVISTA SABERES APUDEP & Volumen 3 Número 2 \\
\hline
\end{tabular}

Sobre la Teoría del Dolo, Claus Roxin ha señalado lo siguiente:

"La diferencia entre el autor [Urheber] y el cómplice sólo puede encontrarse en la independencia de la voluntad de autor y la dependencia de la de cómplice. El cómplice quiere el resultado sólo para el caso de que el autor lo quiera y, para el caso de que el autor no lo quiera, él tampoco lo quiere. Por tanto, debe dejar a criterio del autor la decisión de si el resultado debe producirse o no" (Roxin, 2015)

De lo anterior se desprende que el autor tendrá una voluntad independiente al momento de cometer el delito. Por ejemplo, el policía B planifica el apoderamiento de paquetes de droga custodiados por el policía A. B convence a A que los hurte y se los entregue. Es $B$ quien tiene una voluntad propia (independiente) mientras que la voluntad de $A$ depende de lo que diga $B$ (dependiente). El policía $B$ depende de lo que le diga el policía $A$. Si B se desaparece, lo más probable es que $A$ no sepa cómo sacarle provecho a la droga sin exponerse tanto. Este ejemplo demuestra que:

"el cómplice sólo puede poseer una voluntad dependiente de la del autor, es decir, que somete su voluntad a la del autor de tal manera que deja a criterio de este si el hecho ha de llegar a la consumación o no. En contraposición a esta voluntad dependiente del cómplice, el coautor no reconoce o acepta una voluntad que domine la suya." (Roxin, 2015)

Frente a la teoría del dolo está la Teoría del Interés, según la cual "la voluntad de autor se caracterizaría por el interés propio en el hecho y la del partícipe por la falta de tal interés." (Roxin, 2015). En este sentido, quien tenga el interés de que el hecho se cometa, entonces será autor. Mientras que, si la persona no tiene interés, pero aun así interviene, entonces es partícipe. La pregunta que se debe hacer el juez es: ¿a quién le interesaba que el hecho se cometiera? El imputado que mejor encaje en esa pregunta, entonces será el autor. 


\begin{tabular}{|c|c|c|}
\cline { 2 - 3 } & REVISTA SABERES APUDEP & Volumen 3 Número 2 \\
\hline
\end{tabular}

Si bien es cierto estas teorías han sido superadas, actualmente existen elementos importantes que pueden ser utilizados a la luz de las modernas corrientes del Derecho penal. Dentro de la teoría del dolo "el criterio de la 'subordinación de la voluntad, puede contemplarse como precursora de la teoría del dominio del hecho." (Roxin, 2015). Esto se debe a que los partícipes al subordinar su voluntad a la del autor “(deben) 'dejar a su criterio' la comisión del hecho, porque es él el que decide sobre la ejecución y, con ello, posee el dominio del hecho." (Roxin, 2015)

En cuanto a la teoría del interés, según Roxin "no hay camino que conduzca a la teoría del dominio del hecho." (Roxin, 2015) Pero su importancia radica en que la jurisprudencia alemana actual "sigue viendo en el interés propio un indicio importante (junto a otros) para la determinación de la autoría" (Roxin, 2015). También, habría autoría si "la voluntad del autor se identifica con el interés en la ejecución del delito." (Gill, 2014).

Ambas teorías (del dolo y del interés) presentan problemas con las principales teorías sobre el Derecho penal vigentes. Esto se debe a que "autoría y participación son manifestaciones o forma de aparición del injusto." (Roxin, 2015). Concepción que con el causalismo era diferente.

Por otro lado, los inconvenientes de la teoría subjetiva son "las dificultades probatorias de los elementos subjetivos y el inconveniente de abrir en exceso la puerta a la arbitrariedad judicial.” (Roca, 2012). ¿Podría un juez penal saber lo que pasaba por la mente de los intervinientes en un delito? De seguro, no podría hacerlo en todos los casos. Por lo que muchas decisiones serían a la arbitrariedad.

Además, esta teoría no puede estar vigente, ya que la misma dependía de una teoría del delito que ya ha sido superada, según la cual los elementos subjetivos se analizaban en la culpabilidad (en la actualidad existen elementos subjetivos que se estudian en la tipicidad, como lo son el dolo y la culpa). 


\begin{tabular}{|c|c|c|}
\cline { 2 - 3 } & REVISTA SABERES APUDEP & Volumen 3 Número 2 \\
\hline
\end{tabular}

En este sentido se ha dicho que "actualmente superada en la práctica totalidad de las legislaciones modernas" (Blanco, 2005).

\section{TEORÍAS MATERIALES}

Las teorías materiales objetivas son "un conjunto importante de concepciones teóricas que pretende delimitar la cualidad de autor y de partícipe." (Gill, 2014). Cabe destacar que no se puede afirmar que la teoría material objetiva es equivalente a la teoría del dominio del hecho. Esta última es una teoría distinta.

Las teorías materiales objetivas "consideran autor a quien realiza la contribución objetiva de mayor importancia al hecho" (Jiménez, 2017). Es decir, aquella persona que objetivamente han aportado más en la ejecución del delito. Se consideran materiales porque buscan "un criterio material situado más allá de la remisión a la descripción típica" (Jiménez, 2017). Mientras que se consideran objetivas porque "recurren a criterios distintos a la actitud interna del sujeto frente a su contribución al hecho" (Jiménez, 2017). De esta manera, esta teoría da por superada la concepción subjetiva.

La teoría material delimitaba a la autoría de la participación de acuerdo a los siguientes criterios:

“a) Hay autoría cuando la actividad del sujeto es físicamente condicionante para la ejecución del hecho.

b) Por contra, hay participación cuando la conducta del sujeto es psíquicamente condicionante de la comisión" (Blanco, 2005)

De lo anterior se desprende que, si el interviniente es condición para que el delito se cometa, entonces será autor, mientras que, si solo es condición psíquica para el delito, entonces es partícipe. Esto es perfectamente válido para la instigación, pero para la complicidad presenta algunos inconvenientes, ya que existen aportes físicos que realiza el cómplice. 


\begin{tabular}{|c|c|c|}
\cline { 2 - 3 } & REVISTA SABERES APUDEP & Volumen 3 Número 2 \\
\hline
\end{tabular}

De manera sintética, la doctora Virginia Arango ha señalado que estas teorías:

"se basan en el elemento material, como también en la noción de "causalidad" en el actuar del sujeto que podrá ser autor o partícipe luego de distinguir entre la conducta de ambos, donde dependiendo de su fundamentación la incidencia causal del autor será determinante, mientras que la del partícipe no será una causa sino más bien condición." (Arango, 2017)

Por lo que una visión de las teorías materiales objetivas es que supera el concepto de causalidad. De esta manera se abre paso el concepto de condición. Tienen el gran mérito de buscar en el plano material y real elementos que sirvan para diferenciar a la autoría de la participación.

\section{TEORÍA DEL DOMINIO DEL HECHO}

La última teoría que se explicará es la del dominio del hecho (Tatherrschaftslehre) que es la dominante en la doctrina penal. La teoría del dominio del hecho "es un desarrollo de enfoques tanto subjetivos como objetivos" (Roxin, 2015). Por lo que cuenta con elementos objetivos y subjetivos para diferenciar ambas categorías. "Halló su primera formulación en el año 1933 en Lobe [...y] Welzel desarrollo después, en el año 1939, el concepto de "dominio del del hecho"' (Roxin, 2015). Aunque solamente Gallas “intentó determinar por primera vez de forma mucho más concreta” (Roxin, 2015).

Por lo que la teoría del dominio del hecho entiende que la autoría es una "realización del tipo en sentido material." (Roxin, 2015). De esta manera se pretende superar la teoría subjetiva, ya que para delimitar la autoría de la participación no se debe preguntar ¿quién tenía ánimo de autor? (teoría subjetiva), sino que se debe preguntar ¿quién controló el hecho? La primera debe buscar su respuesta en la intención, con todas sus dificultades probatorias, mientras que la segunda (dominio del hecho) busca su respuesta en la realidad material. 


\begin{tabular}{|c|c|c|}
\cline { 2 - 3 } & REVISTA SABERES APUDEP & Volumen 3 Número 2 \\
\hline
\end{tabular}

Se debe aclarar que esta teoría tiene varias corrientes, por lo que se explicará inicialmente la dominante, y luego se pasará a las demás. La doctrina dominante sobre el dominio del hecho señala que:

"la figura central del suceso delictivo es quien domina el acontecer que conduce a la realización del delito, mientras que los partícipes, sin bien ejercen igualmente influencia en el acontecer, sin embargo, no configuran de manera decisiva 0 determinante su ejecución." (Roxin, 2015).

Es decir, aquel interviniente en un delito que tenga la capacidad de controlar las decisiones directamente vinculadas al delito será considerado autor; mientras que el interviniente que no tenga poder de decidir en la ejecución del delito, entonces será considerado partícipe (que puede ser cómplice o instigador). Otra manera de decirlo es que, según esta teoría, "el dominio del hecho se debe manifestar en una configuración del suceso y quien no sabe que tiene tal configuración real en sus manos carece del dominio del hecho." (Gill, 2014) En este mismo sentido se ha dicho que:

"el que tenga el dominio del hecho será autor, aunque no realice un elemento del tipo (autor mediato o coautor), siendo la voluntad el factor que impulsa y configura la acción, "si se tiene el dominio de la voluntad, se tiene el dominio de la acción" (Benítez, 2007).

De lo anterior se puede deducir que no se requiere que el interviniente realice la conducta personalmente para que sea sancionado como autor del delito, sino que se exige que este tenga un control sobre la realización del delito. Esto permite ser más respetuoso de las categorías ónticas o materiales; o lo que es lo mismo lo que la realidad refleja.

Como se ha dicho previamente, la teoría del dominio del hecho "adolece de una formulación totalmente unificada" (Blanco, 2005). Sino que existen diversas formas de entender esta teoría. Dichas formas son las siguientes: 


\begin{tabular}{|c|c|c|}
\cline { 2 - 3 } & REVISTA SABERES APUDEP & Volumen 3 Número 2 \\
\hline
\end{tabular}

Está la tesis del plan de la voluntad desarrollada por Welzel según la cual "autor del delito "es sólo aquel que, mediante la dirección consciente y final del curso causal hacia el resultado típico, es señor sobre la realización del tipo" (Jiménez, 2017). Esto se debe a que esta tesis trae un concepto de autor "basado precisamente en el concepto final de acción" (Del Castillo, 2007). Esto se debe a que fue precisamente Welzel el creador del finalismo como teoría sustentadora del Derecho penal.

También está la tesis de la comisión directa desarrollada por Gallas, requiere que "el sujeto tenga en sus manos el hecho" (Blanco, 2005). Es decir, no basta con que el interviniente quiera para sí la comisión del delito, sino que efectivamente tenga un control sobre el mismo. Sin embargo, esta tesis presente grandes dificultades para penalizar la autoría mediata, ya que en esta el autor no actúa directamente, sino que lo hace a través de un tercero, que sería un mero instrumento.

Por otro lado, está la tesis de la distribución real de fuerzas de Maurach y Gössel, según la cual es autor "el interviniente en la infracción penal que puede voluntariamente, conforme a la real distribución de fuerzas entre los diversos sujetos concurrentes, impedir o dejar que se perfeccione la ejecución del hecho." (Blanco, 2005). El problema radica en que puede existir un control psicológico sobre el hecho (autoría mediata y coautoría) que no pueda ser medido bajo la premisa de la fuerza, y menos de la fuerza física.

Todas las tesis anteriores presentan problemas al momento de delimitar los casos prácticos, especialmente aquellos en los cuales los intervinientes no actúen directamente. Finalmente, está la tesis de los ámbitos, propuesta por Claus Roxin, según la cual hay que distinguir tres formas de autoría: dominio de la acción, dominio de la voluntad y dominio funcional. 


\begin{tabular}{|c|c|c|}
\cline { 2 - 3 } & REVISTA SABERES APUDEP & Volumen 3 Número 2 \\
\hline
\end{tabular}

El dominio de la acción sirve para fundamentar la autoría ya que "de su comportamiento se desprenda el hecho de que este no estaba bajo ninguna coacción o bajo el mando de otra persona, que toda realización del tipo que este logre siempre estuvo supeditada a su propia voluntad" (Arango, 2017). Por ejemplo, cuando A hurta un vehículo, es él mismo quien domina voluntariamente toda la acción, y, por ende, es autor.

El dominio de la voluntad sirve para fundamentar la autoría mediata que es cuando "una persona se vale de otro para lograr un resultado que es típico, sea a través de error o por un estado de necesidad." (Arango, 2017). Es decir, una persona puede estar consciente que está cometiendo un delito, pero aun así ser instrumento del autor mediato. Por ejemplo, A secuestra a B y su familia, a quienes amenaza con matarlos, para que $B$ vaya a su oficina y se apropie de unos secretos comerciales. En este supuesto, el que tenga la intención de apropiarse ilícitamente de los documentos será autor, pero como utiliza a otra persona, entonces, a través de otro ejerce su propia voluntad, por lo que $A$ es autor mediato.

El dominio funcional del hecho fundamenta la coautoría en la cual "varios sujetos se dividen la ejecución o preparación de un hecho delictivo." (Arango, 2017). Por ejemplo, A y $B$, en común acuerdo, deciden robar en un negocio, por lo que, $A$ amenaza a los presentes con un arma, mientras que $B$ toma sus pertenencias. En este supuesto hay una dominio funcional y no total del delito por parte de los intervinientes. Con base en lo anterior, A y B deben ser sancionados como coautores.

Se puede finalizar este punto, señalando que la teoría del dominio del hecho ha venido "a precisar con mayor nitidez el concepto de autoría, llevándola más allá de la simple ejecución del hecho a otras formas de realización del mismo, bien juntamente con otros, bien por medio de otro sirviéndose de él como instrumento." (Muñoz y García, 2010,). Por lo que, de todas las teorías esbozadas hasta este momento por el presente artículo, ésta es la que más se adecúa a criterios normativos y materiales. 


\begin{tabular}{|c|c|c|}
\cline { 2 - 3 } & REVISTA SABERES APUDEP & Volumen 3 Número 2 \\
\hline
\end{tabular}

Para Hipólito Gill, "es necesario una mayor concreción del dominio del hecho para lograr un concepto más operativo" (Gill, 2014). Por lo que la discusión sobre una teoría que delimite la autoría de la participación todavía no se ha agotado. Aunque la teoría del dominio del hecho es la dominante, no se puede negar que presenta algunas dificultades (por ejemplo, frente a delitos imprudentes). Además, esta teoría de origen alemán no ha evitado que los tribunales de su país la utilicen de manera exclusiva.

\section{LA TEORÍA NORMATIVA DE LA COMBINACIÓN DE LA JURISPRUDENCIA ACTUAL ALEMANA}

El Bundesgerichtshof (BGH) o Tribunal Federal de Justicia de Alemania ha desarrollado su propia teoría, conocida como la teoría normativa de la combinación, que es una variación de la teoría subjetiva, con elementos de la teoría del dominio del hecho. Esta teoría parte de que "la voluntad de autor o es "un hecho interior que el juez de instancia (encargado de fijar los hechos probados) pueda constatar de manera vinculante. Se trata más bien de un juicio valorativo." (Roxin, 2015). Esto no es más que una mezcolanza entre la teoría del dominio del hecho, y algunos aspectos de la teoría subjetiva.

Esta valoración se basa "en el grado de interés propio en el resultado del hecho, en la magnitud de la intervención en el hecho y en el dominio del hecho" (Roxin, 2015). Su gran problema estriba en que "los criterios valorativos por los que habría de determinarse la autoría ni se mencionan de forma concluyente ni se colocan en un orden o se someten siquiera a un principio rector." (Roxin, 2015). Esto produce una inseguridad jurídica al momento de delimitar las responsabilidades de los participantes, ya que resulta problemático "cuando alguien actúa con fuerte interés propio, pero sin dominio del hecho, o con dominio del hecho, pero sin especial interés propio." (Roxin, 2015). Estas disyuntivas pueden ser resueltas dándole una mayor preponderancia a la teoría del dominio del hecho, sin embargo, existe la posibilidad de aplicarle criterios subjetivos. 


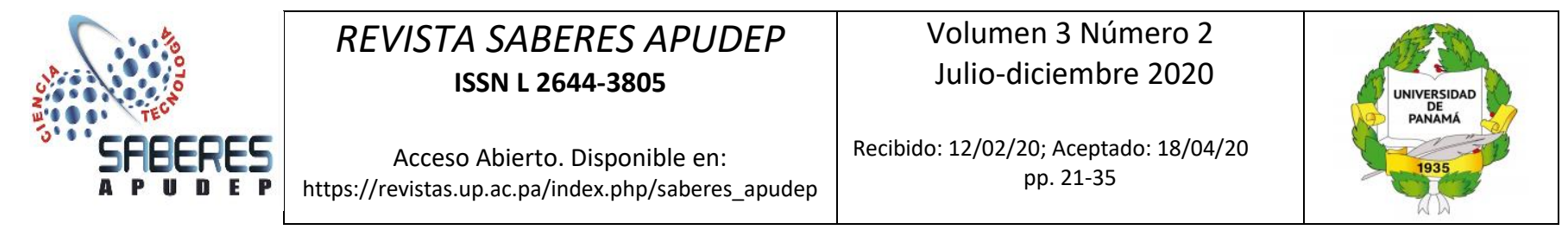

\section{CONCLUSIÓN}

La teoría del dominio del hecho es la dominante en la dogmática penal, sin embargo, la teoría subjetiva no ha sido superada totalmente, por lo que mantiene un nivel de aceptación. Por su parte, las teorías objetivas han sido superadas totalmente.

Las teorías objetivo-formales distinguen entre autor y partícipe sobre la base de quién realice la conducta descrita como típica en la norma penal. Quien realice el verbo rector, será considerado autor. Esta visión ha sido superada.

Las teorías subjetivas distinguen entre autor y partícipe sobre la base de quién quería que el delito se realizara. Es decir, depende de la dirección de la voluntad del interviniente. Con base en lo anterior, es autor quien tiene el animus auctoris, mientras que es partícipe quien tenga el animus socii. Esta teoría tiene presenta un gran problema probatorio, de ahí su poca utilidad.

Las teorías materiales objetivas distinguen entre autor y partícipe sobre la base de quién realiza la contribución material y objetiva de mayor importancia al hecho. Es decir, es autor quien objetivamente ha aportado más en la ejecución del delito, mientras que es partícipe aquel que lo ha hecho en menor medida. Esta teoría fue refinada, y, sirvió de base para la teoría del dominio del hecho.

La teoría del dominio del hecho distingue entre autor y partícipe sobre la base de quién domina el acontecer que conduce a la realización del delito. De lo anterior se desprende que es autor aquel que domina la realización del hecho delictivo, mientras que es partícipe quien no configuran de manera decisiva dicho delito.

El Tribunal Federal de Justicia de Alemania no utiliza de manera exclusiva una única teoría, sino que ha desarrollado una propia, conocida como la teoría normativa de la combinación, que es una variación de la teoría subjetiva, con elementos de la teoría del dominio del hecho. 


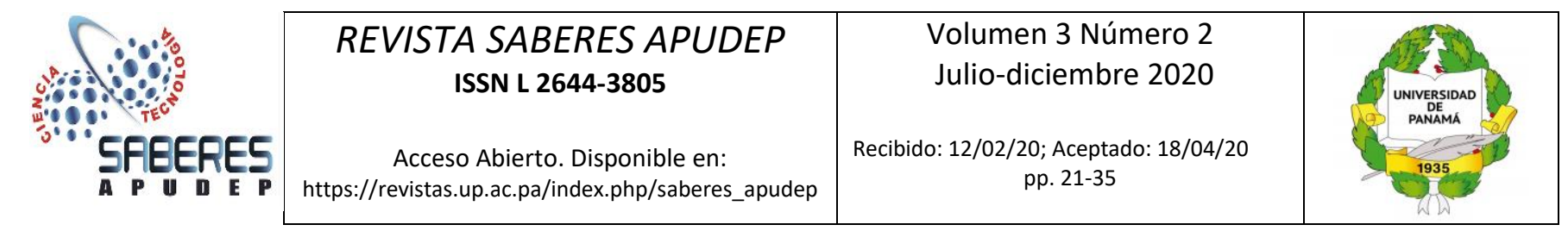

\section{REFERENCIAS BIBLIOGRÁFICAS}

Arango, V. (2017). Derecho penal parte general. Introducción y teoría del delito. $2^{2}$ edición. Panamá: Ediciones Panamá Viejo.

Benítez Ortúzar, I. (2007). La participación en el delito imprudente en el Código Penal español de 1995. Madrid, España: Editorial DYKINSON, S.L.

Blanco, C. (2005). Tratado de Derecho penal español. Tomo I. Volumen 2. La estructura del delito. Barcelona: J. M. Bosch Editor.

Del Castillo Codes, E. (2007). La imprudencia: autoría y participación. Madrid, España. Editorial DYKINSON, S.L.

Escrihuela, J. (2016). Todo penal. Madrid, España: Wolters Kluwer España, S.A.

Gill, H. (2014). Derecho penal (Parte General). 2ª edición. Panamá, Panamá: Imprenta Grafos Litografía.

Jiménez Martínez, C. (2017). Dominio del hecho y autoría mediata en aparatos organizados de poder. Madrid, España: Dykinson S.L.

Miró Llinares, F. (2009). Conocimiento e imputación en la participación delictiva. Aproximación a una teoría de la intervención como partícipe en el delito. Barcelona, España: Atelier Libros Jurídicos.

Muñoz, F. \& García, M. (2010). Derecho Penal Parte General. Valencia, España: Tirant lo Blanch.

Roca De Agapito, L. (2012). Las acciones cotidianas como problema de la participación criminal. Valencia, España: Editorial Tirant lo Blanch.

Roxin, C. (2015). Derecho Penal Parte General. Tomo II. Buenos Aires, Argentina: Thomson Reuters-Civitas. 